Published in final edited form as:

Gynecol Oncol. 2017 May ; 145(2): 224-229. doi:10.1016/j.ygyno.2017.02.036.

\title{
Long-Term Incidence of Female-Specific Cancer after Bariatric Surgery or Usual Care in the Swedish Obese Subjects Study
}

\author{
Åsa Anveden, M.D. ", Magdalena Taube, Ph.D. ${ }^{\star}$, Markku Peltonen, Ph.D. ${ }^{*}$, Peter Jacobson, \\ M.D., Johanna C. Andersson-Assarsson, Ph.D., Kajsa Sjöholm, Ph.D., Per-Arne Svensson, \\ Ph.D., and Lena M.S. Carlsson, M.D.* \\ Department of Molecular and Clinical medicine, Institute of Medicine, the Sahlgrenska Academy \\ at University of Gothenburg (ÅA, MT, PJ, JCAA, PAS, LMSC); Department of Surgery, Hallands \\ hospital, Halmstad, Sweden (ÅA); Department of Chronic Disease Prevention, National Institute \\ of Health and Welfare, Helsinki, Finland (MP) and Institute of Health and Care Sciences, \\ Sahlgrenska Academy at University of Gothenburg (PAS)
}

\section{Summary}

Objective-To examine the long-term effects of bariatric surgery on female-specific cancer in women with obesity.

\begin{abstract}
Methods-The prospective, matched Swedish Obese Subjects (SOS) study was designed to examine outcomes after bariatric surgery. This study includes 1420 women from the SOS cohort that underwent bariatric surgery and 1447 contemporaneously matched controls who received conventional obesity treatment. Age was 37-60 years and BMI was $38 \mathrm{~kg} / \mathrm{m}^{2}$. Information on cancer events was obtained from the Swedish National Cancer Registry. Median follow-up time was 18.1 years (interquartile range 14.8-20.9 years, maximum 26 years). This study is registered with ClinicalTrials.gov, NCT01479452.
\end{abstract}

Results-Bariatric surgery was associated with reduced risk of overall cancer (hazard ratio $=0.71$; 95\% CI 0.59-0.85; $\mathrm{p}<0.001)$. About half of the observed cancers were female-specific, and the incidence of these were lower in the surgery group compared with the control group (hazard ratio $=0.68 ; 95 \%$ CI $0.52-0 \cdot 88 ; \mathrm{p}=0.004)$. The surgical treatment benefit with respect to femalespecific cancer was significantly associated with baseline serum insulin (interaction $\mathrm{p}$

Corresponding author: Per-Arne Svensson, SOS Secretariat, Vita Stråket 15, Sahlgrenska University Hospital, S-413 45 Gothenburg, Sweden., Per-Arne.Svensson@ medic.gu.se, Telephone: +46 (0)733 828386.

*A.A. and M.T. contributed equally to this work

professor

Contributors

PAS, LMSC, MP, and ÅA designed the study. MT and ÅA wrote the paper with contributions from all authors. MP did the statistical analysis and all authors contributed to data interpretation. LMSC, KS, PJ, and JCAA organized register linkage with the Swedish authorities. All authors critically reviewed the content and contributed to manuscript revision, approved the final version and agree to be accountable for all aspects of the work.

Declaration of interests

LMSC has obtained lecture fees from AstraZeneca, Johnson\&Johnson, and MSD. KS holds stocks in Pfizer. No other conflict of interest relevant to this study was reported.

Publisher's Disclaimer: This is a PDF file of an unedited manuscript that has been accepted for publication. As a service to our customers we are providing this early version of the manuscript. The manuscript will undergo copyediting, typesetting, and review of the resulting proof before it is published in its final citable form. Please note that during the production process errors may be discovered which could affect the content, and all legal disclaimers that apply to the journal pertain. 
value $=0.022$ ), with greater relative treatment benefit in patients with medium or high insulin levels. Separate analyses of different types of female-specific cancers showed that bariatric surgery was associated with reduced risk of endometrial cancer (hazard ratio $=0.56$ : $95 \%$ CI $0.35-0.89$; $\mathrm{p}=0.014)$.

Conclusions-In this long-term study, bariatric surgery was associated with reduced risk of female-specific cancer, especially in women with hyperinsulinemia at baseline.

\section{Introduction}

It is well known that obesity is associated with an increased risk of cancer [1-4]. Considering the high obesity prevalence, with more than 600 million people affected globally in 2014 [5], obesity is an important risk factor for cancer. In a recent study, $3.6 \%$ of all new cancer cases in adults were attributable to excess body weight, with a greater fraction in women (5.4\%) than in men (1.9\%) [6]. Obesity is also associated with more advanced cancer disease and increased cancer mortality [7]. In addition, various cancers, including endometrial and pancreatic cancer, are associated with type 2 diabetes, a common co-morbidity related to obesity [8].

Bariatric surgery is the most effective treatment for achieving sustainable weight loss in patients with obesity and it reduces the risk for morbidity and mortality [9-12]. Specifically, bariatric surgery has been shown to reduce the overall cancer risk in patients with obesity [13, 14]. In 2009 we reported that bariatric surgery was associated with reduced incidence of cancer in the Swedish Obese Subjects (SOS) study, an ongoing, non-randomized, prospective, controlled intervention trial investigating long-term effects of bariatric surgery [15]. We found that the cancer-preventive effect of bariatric surgery was seen in women, whereas there was no effect in men; an observation that has also been reported by Adams et al. [13]. In our previous report, the low incidence rate of specific cancers prevented a more detailed analysis. However, in the current study, with a median follow-up time of 18.1 years, we have a larger number of cancer events. The aim of this study was to further investigate the association between bariatric surgery and cancer in women, with focus on femalespecific cancer, i.e. breast cancer and gynaecological cancers.

\section{Methods \\ The SOS study}

The SOS study is an ongoing, prospective matched intervention trial comparing bariatric surgery with conventional obesity treatment [9]. The study has been registered at ClinicalTrials.gov, identifier: NCT01479452. In brief, the study enrolled 4047 patients with obesity recruited through campaigns in mass media and at surgical departments and primary health care centres in Sweden between September 1, 1987 and January 31, 2001. The current analyses include women only. Inclusion criteria were: aged 37-60 years and a BMI >38 $\mathrm{kg} / \mathrm{m}^{2}$. Exclusion criteria, identical in surgery and control groups, were minimal and aimed at obtaining an operable surgical group. Matching of the control group and surgery group was performed on group level using 18 matching variables [16]. The intervention study 
began on the day of surgery for both the surgically treated individual and the matched control.

Seven regional ethics review boards approved the SOS study protocol, and informed consent was obtained from all participants. The primary endpoint of the study was overall mortality, which was reported in 2007 [9]. The secondary endpoints were diabetes [17], gallbladder disease [18], and cardiovascular disease [12]. The outcome of the current study, cancer incidence, was not a predefined endpoint.

\section{Intervention}

Among the 1420 women in the SOS surgery group, 260 (18.3\%) underwent non-adjustable or adjustable gastric banding, $970(68.3 \%)$ underwent vertical banded gastroplasty, and 190 $(13.4 \%)$ underwent gastric bypass. Women in the control group $(n=1447)$ received the conventional treatment for obesity at their primary health care centre, ranging from advanced life-style advice to basically no professional treatment at all.

\section{Examinations}

Surgery and control participants underwent a baseline examination approximately four weeks before the start of intervention. Thereafter clinical examinations were carried out after $0.5,1,2,3,4,6,8,10,15$ and 20 years. Centralized biochemical examinations were carried out at matching and baseline examinations, and after 2, 10, 15 and 20 years. Questionnaires were filled out at every clinical examination.

\section{Data collection}

Baseline characteristics were obtained from the clinical examination, questionnaires and centralized blood chemistry. Baseline alcohol intake was calculated from dietary questionnaires, as previously described [19]. Postmenopausal state was defined as a negative answer to the question: "Do you still menstruate?", or time for surgical menopause. Smoking was defined as a positive answer to the question: "Do you smoke daily?" Data on cancer incidence, death and emigration were obtained by cross-checking social security numbers from the SOS database with the Swedish National Cancer Registry, the Cause of Death Registry and the Registry of the Total Population. The Swedish National Cancer Registry has over $95 \%$ coverage for all malignant tumours of which $99 \%$ are morphologically verified [20]. All malignant tumour diagnoses in the Swedish Cancer Registry were included. Female-specific cancer includes breast, endometrial, ovarian, cervix and all other gynaecological cancers. The cut-off date for the current report was December 31, 2013.

\section{Statistical analysis}

To describe baseline characteristics, mean values and standard deviations were used. All pvalues are two-sided and $\mathrm{p}$ values of less than 0.05 were considered to indicate statistical significance. Statistical analyses were carried out using Stata version 12·1.

Comparisons between the surgery and the control group were done with one-way ANOVA for continuous variables and Fisher's exact test for dichotomous or discrete variables. To 
analyse cancer incidence, Kaplan-Meier estimates of cumulative incidence rates were used to compare time to first cancer diagnosis between different treatment groups. Furthermore, Cox proportional hazards models were used to calculate hazard ratios for surgical treatment for overall cancers and female-specific cancer i.e. breast, ovary, endometrial, cervix and other gynaecological cancers. No adjustments were made for multiple comparisons. To assess the impact of baseline differences between the surgery and control group, analyses were adjusted for baseline confounders; age, BMI and smoking status. The number needed to treat (NNT) to prevent one cancer event during 10 years was estimated by calculating the reciprocal of the absolute risk reduction. A per protocol approach was used in all analyses; thus, all participants were included in their original study group until any bariatric surgery was performed in the control group or there was a change in, or removal of, the bariatric surgical procedure in the surgery group, after which they were censored from the analysis.

In the interaction analysis, the incidence rates were calculated in subgroups defined by risk factors at baseline. The subgroups were based on tertiles of insulin, BMI, blood glucose or glucose status at baseline (normoglycemia, impaired fasting glucose or type 2 diabetes). The influence of bariatric surgery on the incidence of cancer events was tested by including the corresponding interaction term [i.e. product of type of treatment (surgery or control), and the corresponding continuous variable] in the Cox proportional hazard model.

To account for undiagnosed cancer at baseline, separate analyses were performed excluding cancer events occurring during the first three years of the study. In an additional sensitivity analysis, women that had undergone hysterectomy and/or oophorectomy before study start were excluded.

\section{Results}

Baseline characteristics of the 2867 women in the SOS intervention study divided by treatment group are shown in Table 1. After bariatric surgery, mean weight losses were 28.0 $\mathrm{kg}, 21.0 \mathrm{~kg}$ and $21.5 \mathrm{~kg}$ at the 2-, 10- and 15- year follow-up visits, respectively (Figure 1). The mean weight changes in the control group were small. Median follow-up time was 18.1 years (interquartile range 14.8-20.9 years, maximum 26 years).

During follow-up there were 267 first time cancer events in the control group and 205 events in the surgery group. Bariatric surgery was associated with reduced risk of overall cancer (hazard ratio $(\mathrm{HR})=0.71: 95 \% \mathrm{CI} 0.59-0.85 ; \mathrm{p}<0.001)$ and the NNT to prevent one cancer event over 10 years was 31 (Figure 2A). Of the first time cancers, $49 \%$ were female-specific, with 135 events in the control group and 97 events in the surgery group (Figure 2B).

Bariatric surgery was associated with reduced incidence of female-specific cancer $(\mathrm{HR}=0.68,95 \%$ CI $0.52-0.88 ; \mathrm{p}=0.004)$. The NNT to prevent one female-specific cancer event over 10 years was 56 . When adjusted for age, BMI and smoking the HR was almost identical (HR=0.68, 95\% CI 0.52-0.89; $\mathrm{p}=0.006)$. Also after the exclusion of cancers that occurred during the first 3 years, the HR was virtually unchanged (Figure S1 in the Supplementary Appendix). 
Interactions between risk factors and treatment are shown in Figure 3. The surgical treatment benefit with respect to female-specific cancer was significantly associated with baseline serum insulin (interaction $\mathrm{p}$ value $=0.022$ ), with greater relative treatment benefit in participants with medium (hazard ratio $=0.62 ; 95 \%$ CI 0.39-0.97; $\mathrm{p}=0.036)$ or high $(0.57$; 95\% CI 0.38-0.86; $\mathrm{p}=0.007$ ) insulin levels, while there was no interaction with baseline BMI ( $\mathrm{p}=0.671$ ). Exclusion of all cancer events that occurred during the first 3 years of follow-up did not affect the outcome of the interaction analysis (Figure S2).

Separate analyses of different female specific cancers showed that breast- and endometrial cancers were the most common female-specific cancer types in the SOS cohort. For all types of female-specific cancer, except for cervix cancer, the incidence rates were lower in the surgery group compared to the control group (Figure 4), but the difference was only significant for endometrial cancer $(\mathrm{HR}=0.56$ : $95 \%$ CI $0.35-0.89 ; \mathrm{p}=0.014)$. In sensitivity analyses, excluding all cancer events during the first 3 years of follow-up, similar results were obtained (Figure S3). Furthermore, the results were also practically the same when women who had undergone hysterectomy and/or oophorectomy were excluded from the analysis (Figure S4).

\section{Discussion}

The association between obesity and cancer is well established and the previously reported protective effect of bariatric surgery on overall cancer in women [21] is confirmed in this report. The main finding of this study is that bariatric surgery also is associated with reduced risk of female-specific cancer, here defined as breast, endometrial, ovarian, and all other gynaecological cancers, $(\mathrm{HR}=0.6895 \% \mathrm{CI} 0.52-0.88, \mathrm{p}=0.004)$. The association between bariatric surgery and female-specific cancer is noteworthy as these comprised about half of the observed cancer events in our cohort and are common in the obese population [1,2,4]. For example, in the UK, as much as $40.8 \%$ of endometrial cancer has been attributed to obesity [1].

In the interaction analysis we found that bariatric surgery was associated with a greater risk reduction of female-specific cancer in women with medium or high insulin levels at baseline compared to those with low insulin levels. Insulin most likely favours cancer development as it is a growth factor with known metabolic and mitogenic effects, and hyperinsulinemia is one of the factors suggested to explain the link between obesity and cancer [8]. In addition insulin is connected to endocrine risk factors for cancer such as insulin-like growth factor 1, sex steroids and adipokines [7], and it has been shown that insulin levels are reduced after bariatric surgery [17].

As the rate of obesity increases, so does the prevalence of endometrial cancer in developed countries, even in young, premenopausal women [22, 23]. In our study, endometrial cancer was one of the most common cancers and when we analysed female-specific cancer types individually we found that bariatric surgery was associated with reduced risk for endometrial cancer $(\mathrm{HR}=0.56$ : $95 \%$ CI $0.35-0.89 ; \mathrm{p}=0.014)$. The preventive effect of bariatric surgery on endometrial cancer has also been observed in a retrospective cohort study by Ward et al. [24]. In addition, in two recent studies, where endometrial biopsies from women with severe

Gynecol Oncol. Author manuscript; available in PMC 2018 May 01. 
obesity were examined before and after gastric bypass surgery, endometrial pathology was decreased after the surgical treatment $[25,26]$. Together with our prospective data, this suggests that bariatric surgery may have a role in prevention of endometrial cancer. Among survivors of endometrial cancer, patients who also have morbid obesity have increased risk of death due to cardiovascular disease $[27,28]$. We have earlier shown that bariatric surgery also is associated with reduced incidence of cardiovascular events [12]. Hence, women with obesity undergoing bariatric surgery may have beneficial effects both on cardiovascular and endometrial cancer risks.

The SOS study has several limitations. For ethical reasons, due to high post-operative mortality during bariatric surgery at the time when the SOS study was started, it could not be randomized. Also, the majority of the participants in our surgery group have undergone vertical banded gastroplasty or banding, methods that are rarely used today. Due to lack of power we cannot exclude the possibility that there are differences between the effects of gastric bypass and these older restrictive procedures. Another limitation is that cancer incidence was not a predefined endpoint in the study, and the study was therefore not designed to address the current research question. There was no formal power calculation for cancer outcome, and all the analyses, including those on specific cancer types are explorative and the results therefore need to be confirmed by other studies. It should be noted that the number of some specific cancer types was low, and non-significant findings need to be interpreted with care. Ideally our results should be confirmed in larger randomized controlled trials with long-term follow-up, designed to examine cancer incidence as a primary endpoint. However, it is questionable whether this would be possible to accomplish or even ethical considering the great benefits from surgery on several other serious outcomes, including cardiovascular events and type 2 diabetes $[11,12]$. Another limitation of our study is that no adjustments were made for multiple comparisons. However, to reduce the risk of random findings, we performed several sensitivity analyses that confirmed the results from the primary analysis, indicating robustness of data. Strengths of the SOS study include the prospective design, the matched control group, long follow-up and the possibility to cross-check social security numbers of participants with national registers that are almost $100 \%$ complete.

In conclusion, our results show that bariatric surgery is associated with reduced incidence of female-specific cancer, and reduced risk of endometrial cancer. The treatment benefit of bariatric surgery is greatest in women with hyperinsulinemia.

\section{Supplementary Material}

Refer to Web version on PubMed Central for supplementary material.

\section{Acknowledgments}

Funding This project was supported by grants from the National Institute of Diabetes and Digestive and Kidney Diseases of the National Institutes of Health under Award Number R01DK105948 (the content is solely the responsibility of the authors and does not necessarily represent the official views of the National Institutes of Health), the Swedish Research Council K2013-99X-22279-01, K2013-54X-11285-19, Sahlgrenska University Hospital ALF research grant and Swedish Diabetes Foundation. 
We thank the staff members at the SOS Secretariat and at 480 primary health-care centres and 25 surgical departments in Sweden that participated in the study. This project was supported by grants from the National Institute of Diabetes and Digestive and Kidney Diseases of the National Institutes of Health under Award Number R01DK105948 (the content is solely the responsibility of the authors and does not necessarily represent the official views of the National Institutes of Health), the Swedish Research Council K2013-99X-22279-01, K2013-54X-11285-19, Sahlgrenska University Hospital ALF research grant and Swedish Diabetes Foundation.

\section{Role of the funding source}

The funders of the study had no role in study design, data collection, data analysis, data interpretation, or writing of the report. PAS, LMSC and MP had full access to all the data. PAS had final responsibility for the decision to submit for publication.

\section{References}

1. Bhaskaran K, Douglas I, Forbes H, dos-Santos-Silva I, Leon DA, Smeeth L. Body-mass index and risk of 22 specific cancers: a population-based cohort study of 5. 24 million UK adults. Lancet. 2014; 384:755-65. [PubMed: 25129328]

2. Calle EE, Rodriguez C, Walker-Thurmond K, Thun MJ. Overweight, obesity, and mortality from cancer in a prospectively studied cohort of US adults. N Eng J Med. 2003; 348:1625-38.

3. Reeves GK, Pirie K, Beral V, Green J, Spencer E, Bull D. Cancer incidence and mortality in relation to body mass index in the Million Women Study: cohort study. BMJ. 2007; 335:1134. [PubMed: 17986716]

4. Renehan AG, Tyson M, Egger M, Heller RF, Zwahlen M. Body-mass index and incidence of cancer: a systematic review and meta-analysis of prospective observational studies. Lancet. 2008; 371:56978. [PubMed: 18280327]

5. Ng M, Fleming T, Robinson M, Thomson B, Graetz N, Margono C, et al. Global, regional, and national prevalence of overweight and obesity in children and adults during 1980-2013: a systematic analysis for the Global Burden of Disease Study 2013. Lancet. 2014; 384:766-81. [PubMed: 24880830]

6. Arnold M, Pandeya N, Byrnes G, Renehan AG, Stevens GA, Ezzati M, et al. Global burden of cancer attributable to high body-mass index in 2012: a population-based study. Lancet Oncol. 2015; 16:36-46. [PubMed: 25467404]

7. De Pergola G, Silvestris F. Obesity as a major risk factor for cancer. J Obes. 2013; 2013:291546. [PubMed: 24073332]

8. Johnson JA, Carstensen B, Witte D, Bowker SL, Lipscombe L, Renehan AG. Diabetes and cancer (1): evaluating the temporal relationship between type 2 diabetes and cancer incidence. Diabetologia. 2012; 55:1607-18. [PubMed: 22476947]

9. Sjöstrom L, Narbro K, Sjöstrom CD, Karason K, Larsson B, Wedel H, et al. Effects of bariatric surgery on mortality in Swedish obese subjects. N Engl J Med. 2007; 357:741-52. [PubMed: 17715408]

10. Adams TD, Gress RE, Smith SC, Halverson RC, Simper SC, Rosamond WD, et al. Long-term mortality after gastric bypass surgery. N Engl J Med. 2007; 357:753-61. [PubMed: 17715409]

11. Carlsson LM, Peltonen M, Ahlin S, Anveden A, Bouchard C, Carlsson B, et al. Bariatric surgery and prevention of type 2 diabetes in Swedish obese subjects. N Engl J Med. 2012; 367:695-704. [PubMed: 22913680]

12. Sjöstrom L, Peltonen M, Jacobson P, Sjöstrom CD, Karason K, Wedel H, et al. Bariatric surgery and long-term cardiovascular events. JAMA. 2012; 307:56-65. [PubMed: 22215166]

13. Adams TD, Stroup AM, Gress RE, Adams KF, Calle EE, Smith SC, et al. Cancer incidence and mortality after gastric bypass surgery. Obesity. 2009; 17:796-802. [PubMed: 19148123]

14. Christou NV, Lieberman M, Sampalis F, Sampalis JS. Bariatric surgery reduces cancer risk in morbidly obese patients. Surg Obes Relat Dis. 2008; 4:691-5. [PubMed: 19026373]

15. Sjöstrom L, Gummesson A, Sjöstrom CD, Narbro K, Peltonen M, Wedel H, et al. Effects of bariatric surgery on cancer incidence in obese patients in Sweden (Swedish Obese Subjects Study): a prospective, controlled intervention trial. Lancet Oncol. 2009; 10:653-62. [PubMed: 19556163] 
16. Sjöström L, Larsson B, Backman L, Bengtsson C, Bouchard C, Dahlgren S, et al. Swedish obese subjects (SOS). Recruitment for an intervention study and a selected description of the obese state. Int J Obes Relat Metab Disord. 1992; 16:465-79. [PubMed: 1322873]

17. Sjöström L, Lindroos A-K, Peltonen M, Torgerson J, Bouchard C, Carlsson B, et al. Lifestyle, diabetes, and cardiovascular risk factors 10 years after bariatric surgery. N Engl J Med. 2004; 351:2683-93. [PubMed: 15616203]

18. Torgerson JS, Lindroos AK, Naslund I, Peltonen M. Gallstones, gallbladder disease, and pancreatitis: cross-sectional and 2-year data from the Swedish Obese Subjects (SOS) and SOS reference studies. Am J Gastroenterol. 2003; 98:1032-41. [PubMed: 12809825]

19. Svensson PA, Anveden Å, Romeo S, Peltonen M, Ahlin S, Burza MA, et al. Alcohol consumption and alcohol problems after bariatric surgery in the Swedish obese subjects study. Obesity. 2013; 21:2444-51. [PubMed: 23520203]

20. Barlow L, Westergren K, Holmberg L, Talbäck M. The completeness of the Swedish Cancer Register-a sample survey for year 1998. Acta oncol. 2009; 48:27-33. [PubMed: 18767000]

21. Tee MC, Cao Y, Warnock GL, Hu FB, Chavarro JE. Effect of bariatric surgery on oncologic outcomes: a systematic review and meta-analysis. Surg Endosc. 2013; 27:4449-56. [PubMed: 23949484]

22. Ferlay J, Soerjomataram I, Dikshit R, Eser S, Mathers C, Rebelo M, et al. Cancer incidence and mortality worldwide: sources, methods and major patterns in GLOBOCAN 2012. Int J Cancer. 2015; 136:E359-E86. [PubMed: 25220842]

23. Morice P, Leary A, Creutzberg C, Abu-Rustum N, Darai E. Endometrial cancer. Lancet. 2016; 387:1094-108. [PubMed: 26354523]

24. Ward KK, Roncancio AM, Shah NR, Davis M-A, Saenz CC, McHale MT, et al. Bariatric surgery decreases the risk of uterine malignancy. Gynecol Oncol. 2014; 133:63-6. [PubMed: 24680593]

25. Linkov F, Elishaev E, Gloyeske N, Edwards R, Althouse AD, Geller MA, et al. Bariatric surgeryinduced weight loss changes immune markers in the endometrium of morbidly obese women. Surg Obes Relat Dis. 2014; 10:921-6. [PubMed: 25439003]

26. Argenta PA, Kassing M, Truskinovsky AM, Svendsen CA. Bariatric surgery and endometrial pathology in asymptomatic morbidly obese women: a prospective, pilot study. BJOG. 2013; 120:795-800. [PubMed: 23231632]

27. Laskey R, McCarroll M, Gruenigen V. Obesity-related endometrial cancer: an update on survivorship approaches to reducing cardiovascular death. BJOG. 2016; 123:293-8. [PubMed: 26841334]

28. Ward KK, Shah NR, Saenz CC, McHale MT, Alvarez EA, Plaxe SC. Cardiovascular disease is the leading cause of death among endometrial cancer patients. Gynecol Oncol. 2012; 126:176-9. [PubMed: 22507532] 


\section{Highlights}

Bariatric surgery is associated with reduced risk of overall female-specific cancer.

Treatment benefit of bariatric surgery is greatest in women with hyperinsulinemia.

Bariatric surgery is associated with reduced risk of endometrial cancer. 


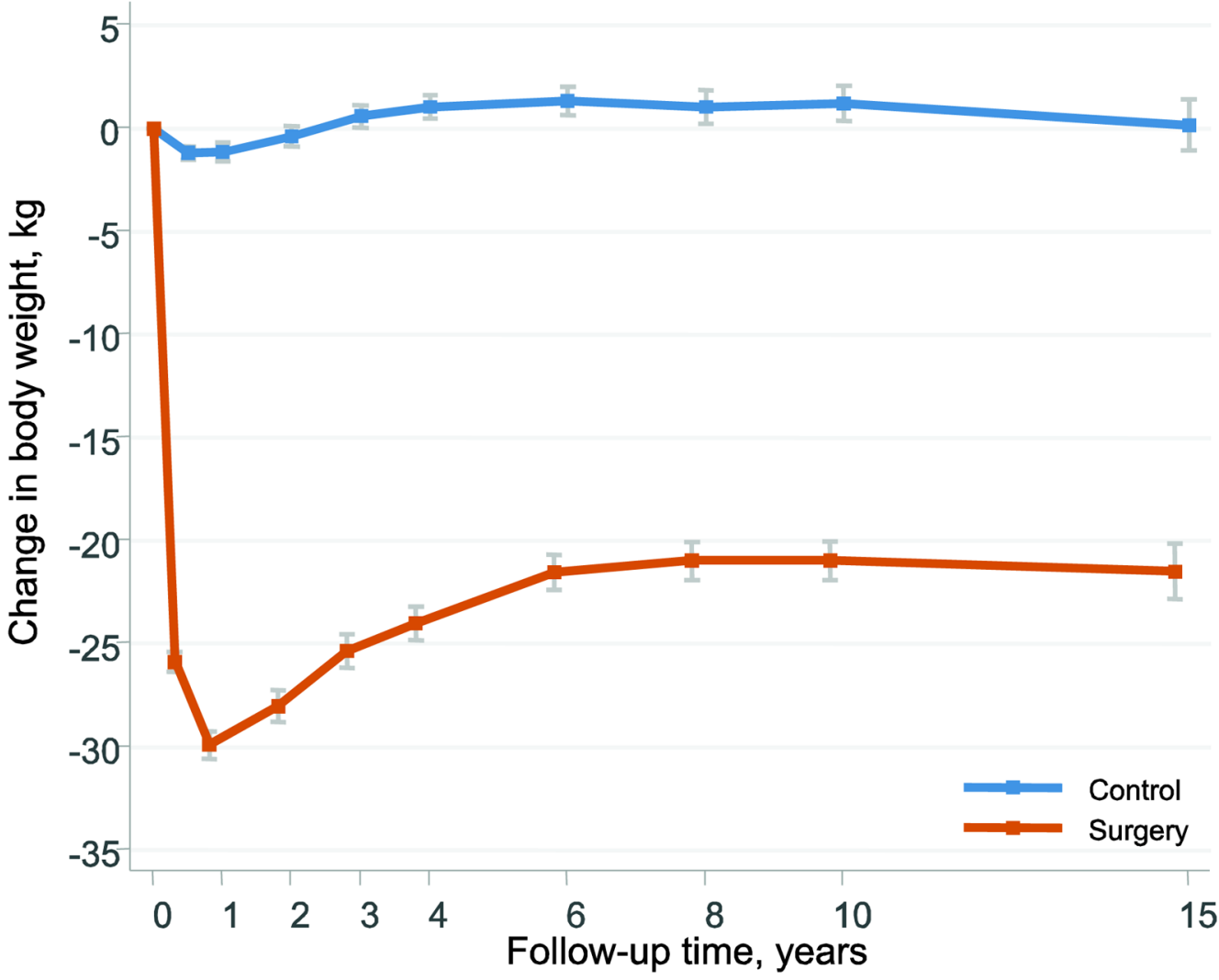

Figure 1. Body weight changes after bariatric surgery or usual care in women of the Swedish Obese Subjects Study

Error bars represent $95 \%$ confidence intervals. 


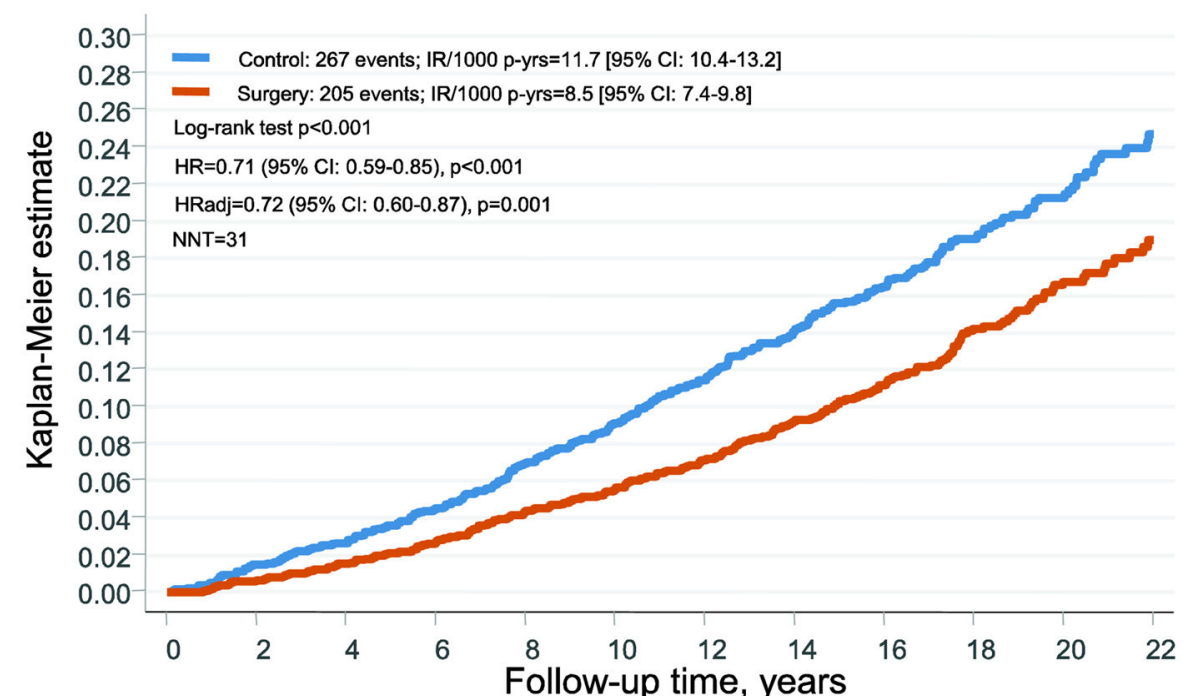

Number at risk

$\begin{array}{lllllllllllll}\text { Control } & 1447 & 1414 & 1379 & 1325 & 1260 & 1189 & 1125 & 977 & 727 & 565 & 365 & 190\end{array}$
Adj: Adjusted for age, baseline BMI and smoking

Fig. $2 \mathrm{~A}$ 


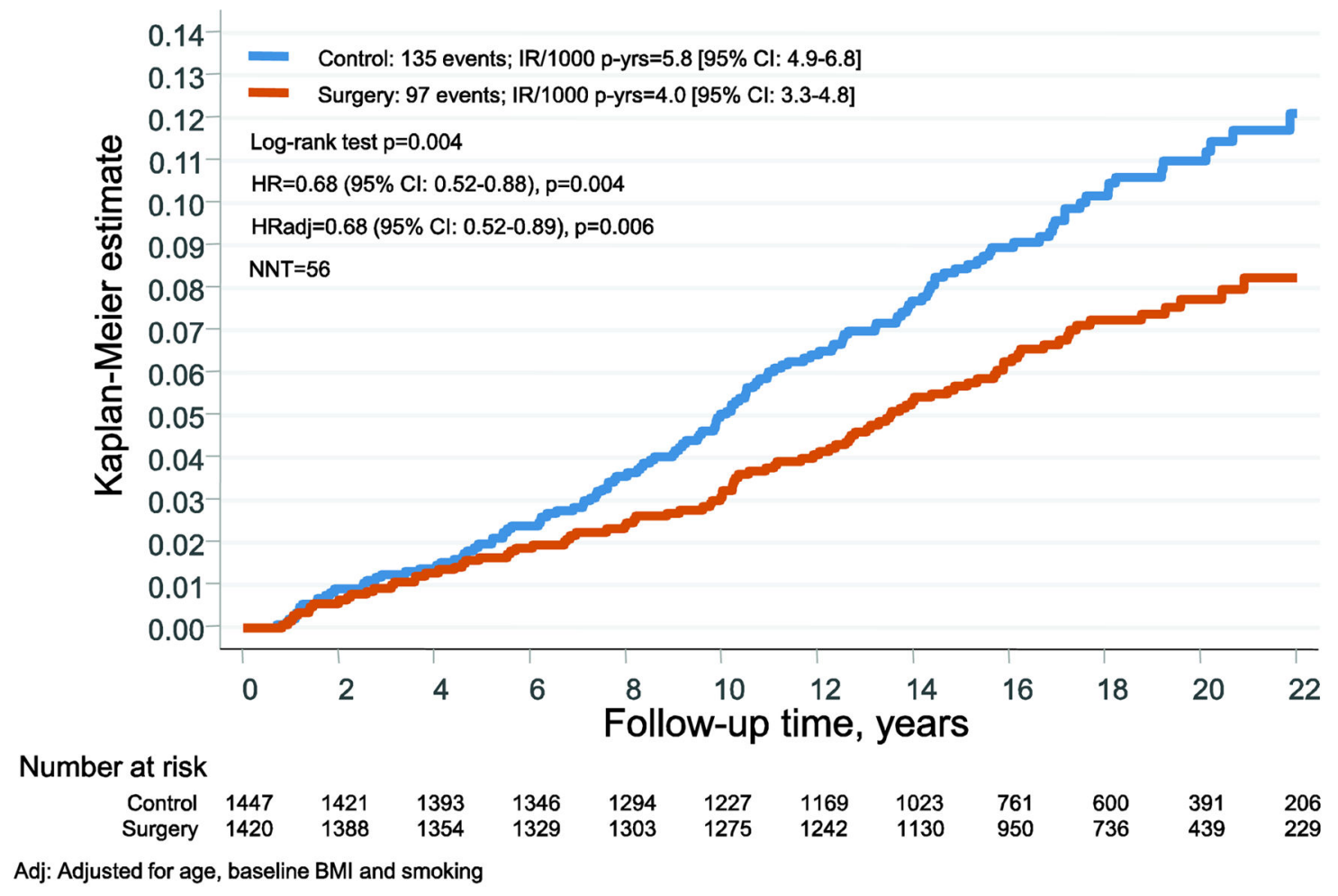

Fig. 2B

Figure 2. Cumulative incidence of cancer events after bariatric surgery or usual care in women of the Swedish Obese Subjects study

(A) overall cancer events and (B) female-specific cancer events. The x-axes are truncated at 22 years but all observations after 22 years were included in the analyses. 


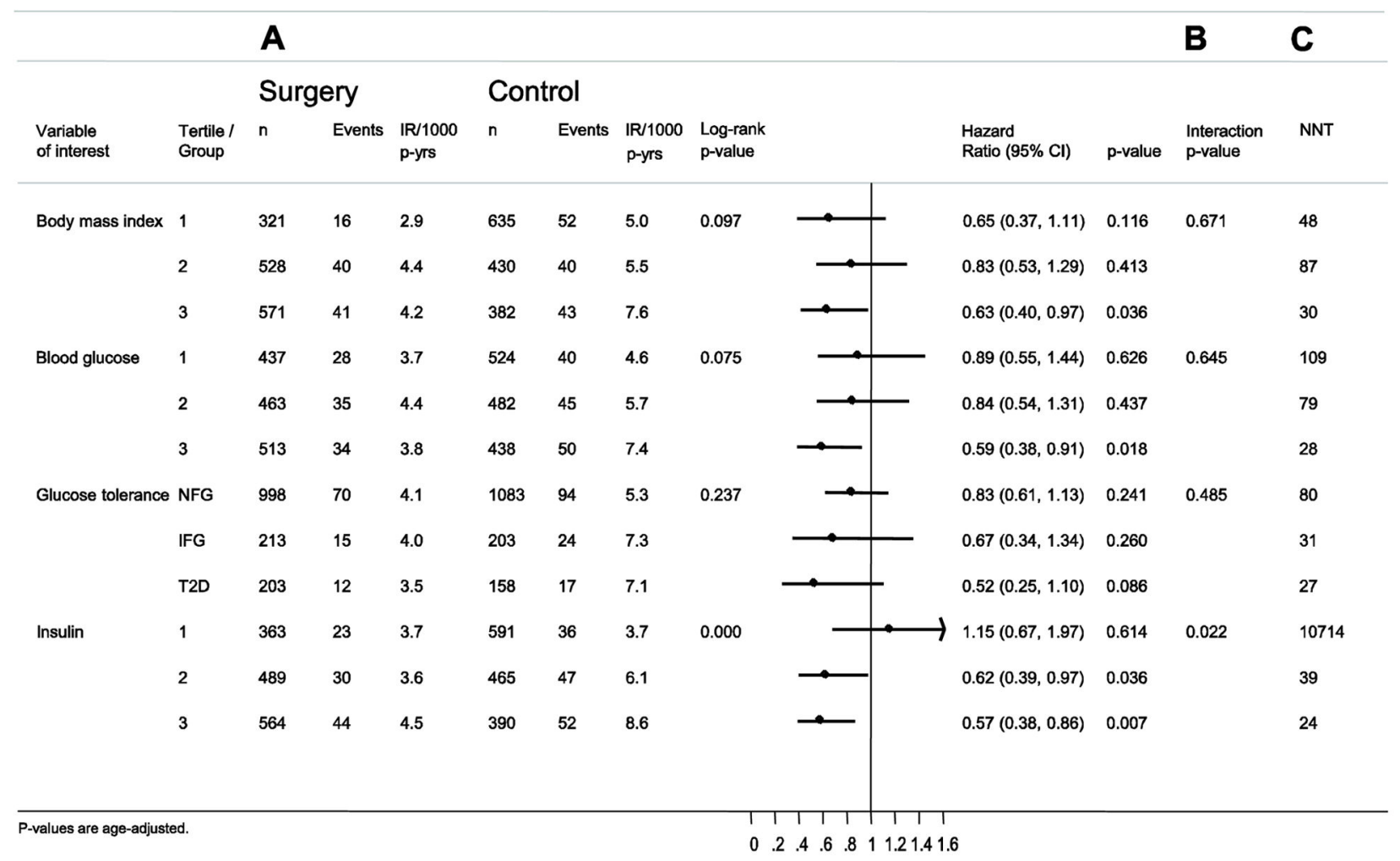

Figure 3. Risk factor-treatment interactions for analyses for incidence of female-specific cancer events in the SOS study

Sub-grouping is based on tertiles of baseline values. (A) Incidence of first time femalespecific cancer events. (B) Risk factor-treatment interactions for female-specific cancer events in subgroups. (C) Number needed to treat (NNT) over 10 years to prevent one female-specific cancer event. 


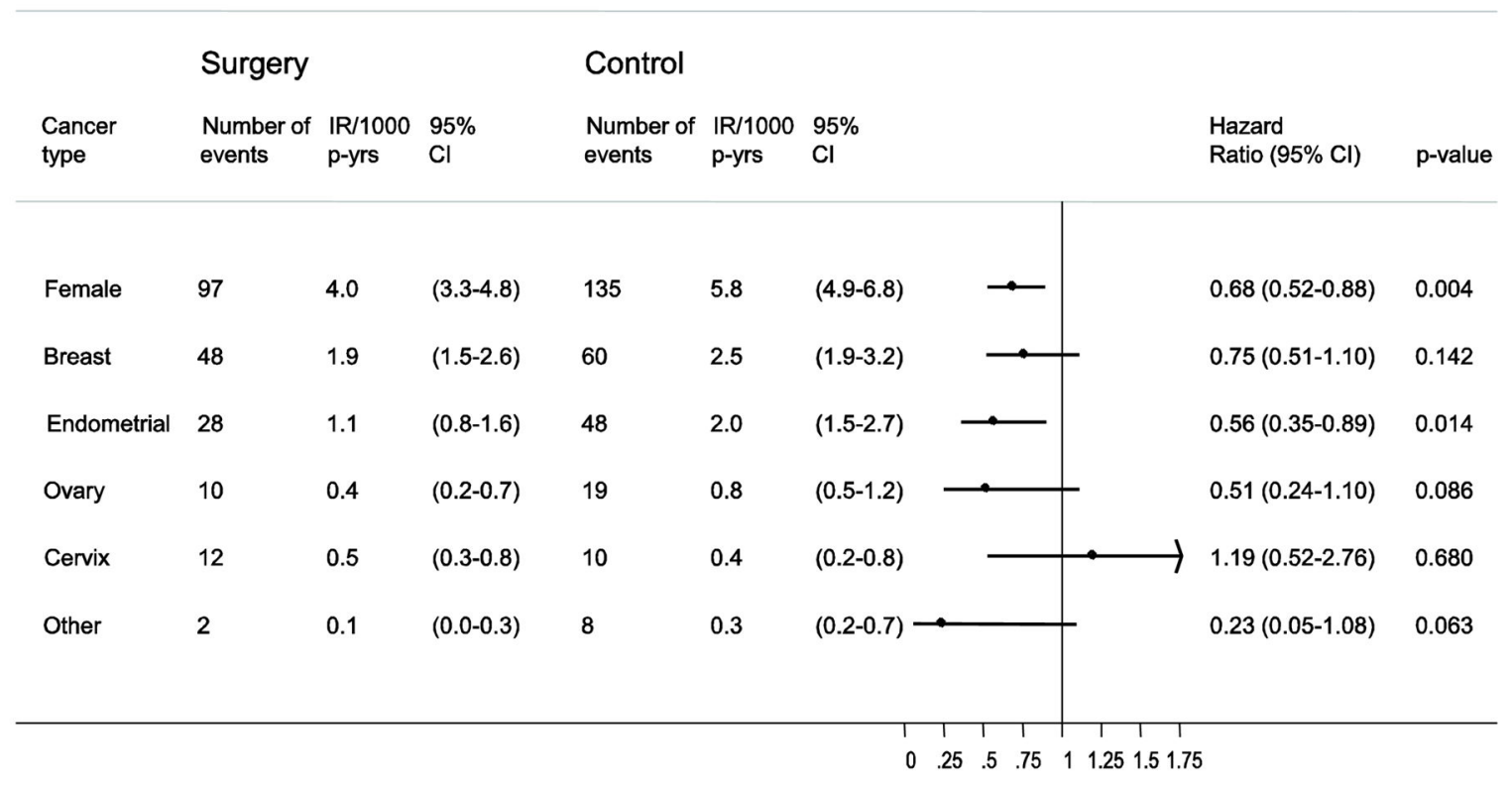

Figure 4. Bariatric surgery and incidence of different types of female-specific cancer Number of events and incidence rates per 1000 person-years (IR/1000 p-yrs) are displayed separately for the surgery and control groups. Hazard ratio (HR) and 95\% CI are shown by bars with dots. 


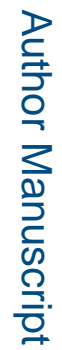

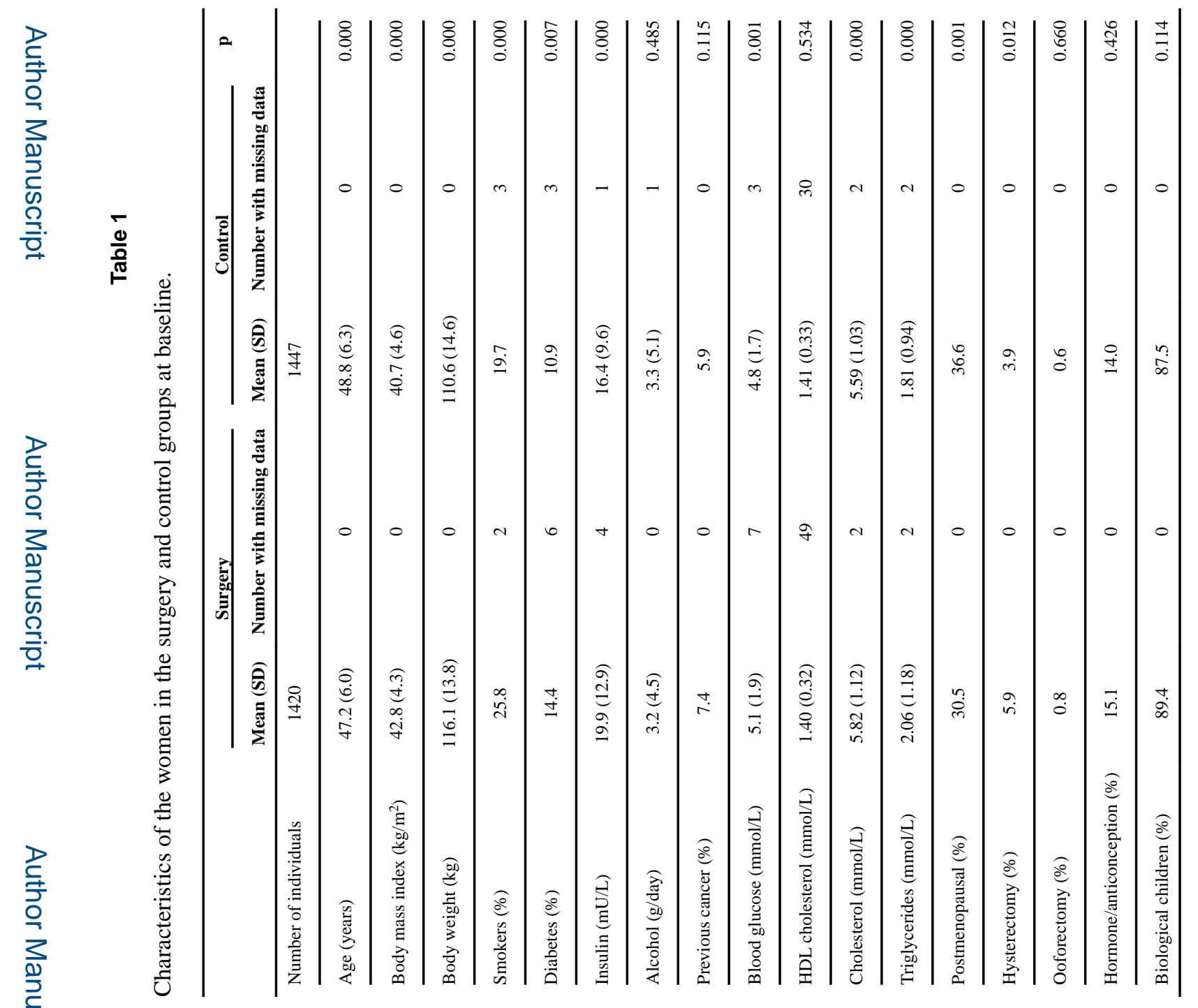

